\title{
ELEMENTS OF STRUCTURE CALCULATION FOR SHAPE MEMORY DEVICE
}

\author{
Y. GILLET, E. PATOOR and M. BERVEILLER \\ Institut Supérieur de Génie Mécanique et Productique, Université de Metz, Ile du Saulcy, F-57045 \\ Metz cedex, France
}

\begin{abstract}
In order to dimension the mechanical elements made of shape memory alloys (SMA), a method based on phenomenological relation between stress and strain has been developed. This method and applications to pseudoelastic helical spring problems are described in this paper.
\end{abstract}

\section{1. - Introduction.}

The use of shape memory alloys (SMA) in industrial applications necessitates to describe their behavior, not only as tensile test specimen in which the stress is homogeneous but as mechanical elements (springs, bearings, beams...) where the stress distribution is, in many cases, inhomogeneous. This problem coupled with the non elastic behavior of SMA leads the classical method of dimensionnement to be inefficient. To overcome this, different approaches have been developed.

First, abacuses have been built, but this route involves to dispose of a collection of data that can be representative in most of the cases that are met (different geometries, materials, thermomechanical loadings) and this approach has no predictive capacity. On the other hand, this problem was the object of many works on the point of view of calculus. Some of them use adapted mechanical engineering classical formulas [1], [2], while the others have taken into account a simplified material behavior (transformation stress considered as constant during the deformation) [3], [4], [5], [6].

The method proposed here, using constitutive equations previously established [7] takes into account a more realistic behavior for SMA. It has been applied on a pure torsion SMA beam and on an helical spring . The results obtained with this pattern are compared to experimental results performed on copper based alloy elements. The parameters used in the theory are deduced from uniaxial tensile tests carried out on the same material.

\section{2. - Inelastic behavior of SMA.}

For a commercial Cu-Zn-AI SMA elaborated by TREFIMETAUX Company, experimental curves in fig. 1 show the displacement $f$ of an helical spring, as a function of the applied load $F$ for different temperatures. It can be noticed that $f$ is not a linear function of $F$, so that mechanical engineering classical formulas cannot be used here. The observation of fig. 2 which shows the stress-strain curve of the same material tested in tension at constant temperature can explain the non-linearity in $f$ by an inelastic behavior of the material which leads to an inhomogeneous stress distribution inside the spring (see fig.3). New mechanical engineering relations must be established taking into account the behavior of SMA. This is performed in this contribution using a transformation criteria (1) and its associated flow rule (2) previously established

[7]. $\sqrt{J_{2}}-\left(\frac{B}{g}\left(T-M_{S}\right)\right)=0$ 


$$
\mathrm{d} \varepsilon^{\mathrm{PT}}=\mathrm{a}\left(\varepsilon^{\mathrm{PT}}\right) \frac{\mathrm{S}_{\mathrm{ij}}}{2 \sqrt{\mathrm{J}_{2}}} \cdot\left(\frac{\mathrm{S}_{\mathrm{hk}}}{2 \sqrt{\mathrm{J}_{2}}} \mathrm{~d} \sigma_{\mathrm{hk}}-\left(\frac{\mathrm{B}}{\mathrm{g}}\right) \mathrm{dT}\right)
$$

Function $\mathrm{a}\left(\varepsilon^{\mathrm{PT}}\right)$ can be identified by developing ( 2 ) considered at constant temperature $(\mathrm{dT}=0)$ for an uniaxial tensile stress field, assuming a stress-strain curve in bilinear form, it comes :

$$
\mathrm{a}\left(\varepsilon^{\mathrm{PT}}\right)=\frac{3}{\mathrm{H}} \quad \text { denoting } \quad \mathrm{H}=\frac{\mathrm{d} \sigma}{\mathrm{d} \varepsilon^{\mathrm{PT}}}
$$

Tangent modulus $\mathrm{H}$ can be easily determined on a tensile curve (fig.2). The knowledge of a( $\varepsilon^{\mathrm{PT}}$ ) allows to determine the material response for another elementary loading. For pure shearing, relation (2) allows to define a shear modulus of transformation $\mu^{\prime}$ defined by

$$
\mu^{\prime}=\frac{d \tau}{d \gamma^{P T}}=\frac{H}{3}
$$

In that way, the different parameters inherent to the material behavior can be determined from measurement on uniaxial tension test (fig. 2).

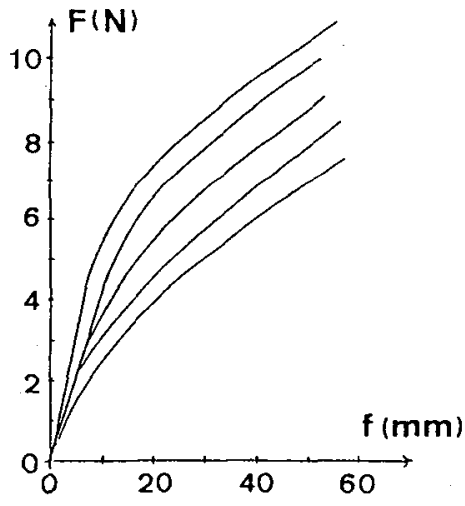

Figure 1: Displacement in function of the applied load at different temperature for a $\mathrm{Cu}-\mathrm{Zn}$-Al Shape Memory helical spring $\left(\mathrm{M}_{\mathrm{S}}=7^{\circ} \mathrm{C}\right)$.

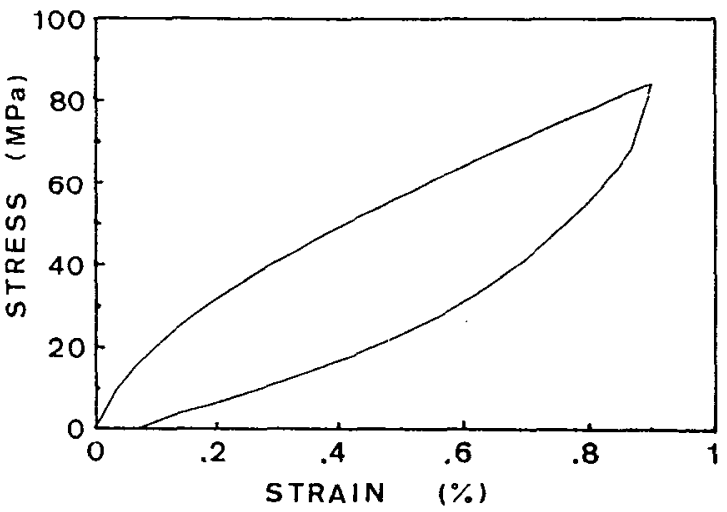

Figure 2: Stress-Strain curve in tension test on the same alloy $\left(\mathrm{M}_{\mathrm{S}}=7^{\circ} \mathrm{C}\right)$ at ambiant temperature for wire diameter $1 \mathrm{~mm}$.

\section{3. - Pure torsion of a cylindrical beam based on the Navier-Bernoulli principle.}

In agreement with the well verified assumption of Navier-Bernoulli, the application of a constant torque to a circular bar of radius $R$ involves a rigid body rotation of the cross section, denoted by $\theta$, about the longitudinal axis, without warping. This gives, at a given radius $\rho$, a displacement vector expressed in cylindrical coordinates by :

$$
u_{x}=0 ; u_{\theta}=\rho \theta ; u_{p}=0
$$

Expressing the deformations in the frame of the infinitesimal strain, and denoting by $\psi$ the angle of twist per unit length, one obtains:

$$
\gamma_{x \theta}(p)=\rho \psi
$$

The stress field $\tau(\rho)$ in a cross section is then determined by using the behavior law of the material, and the expression of the torque $M_{t}$ in function of the twist angle $\psi$ is obtained by using the classical formulae : 


$$
M_{t}=\int_{S} \tau(\rho) \cdot \rho \cdot d S
$$

where $S$ is the area of the cross section. Due to the inelastic behavior of SMA , the stress $\tau(\rho)$ is not related to the twist angle $\psi$ in a simple way. The existence of a stress-induced phase transformation implies to define a critical rotation of transformation (resp. end of transformation) $\psi_{\mathrm{S}}$ (resp. $\psi_{\mathrm{f}}$ ) which is associated to $\gamma_{\mathrm{S}}$ (resp. $\gamma_{\mathrm{f}}$ ) the critical shear strain. These quantities are related to the temperature through criteria (1) and by the Hooke's law.

With respect to the rigid body rotation, the evolution of the transformation leads to consider the existence of untransformed and transformed zones delimited by $R_{a}$ and $R_{m}$ (fig.3) which are defined from (5):

$$
\gamma_{\mathrm{s}}=\psi_{\mathrm{s}} \mathrm{R}=\psi \mathrm{R}_{\mathrm{a}} \quad \text { and } \quad \gamma_{\mathrm{f}}=\psi_{\mathrm{f}} \mathrm{R}=\psi \mathrm{R}_{\mathrm{m}}
$$

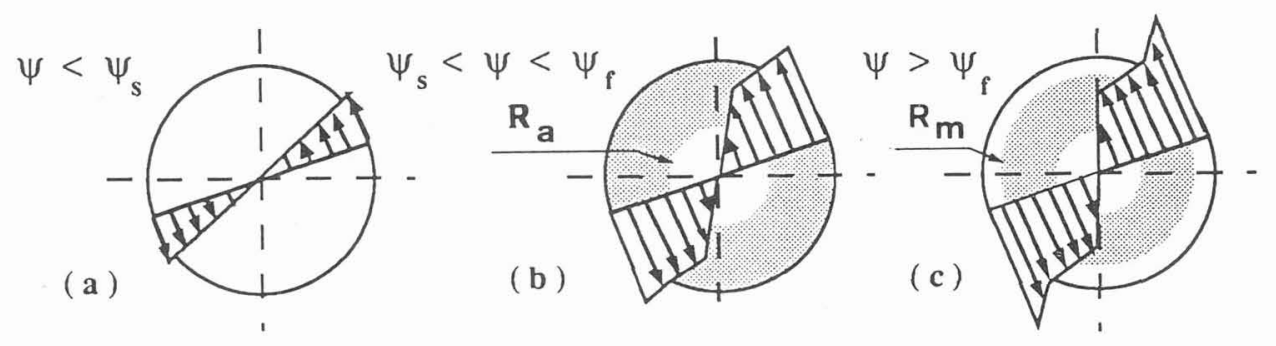

Figure 3: Distribution of shear stress in the cross section of a circular bar in function of the twist angle.

- When $\psi<\psi_{\mathrm{s}}$ (fig. 3a), there is no transformation and classical elastic relations remain valid.

- When $\psi_{s}<\psi<\psi_{\mathrm{f}}$, two zones having a different behavior have to be considered (fig. 3b), an elastic core where the behavior is caracterized by $\mu_{a}$, the elastic shear modulus of austenite, and a transformation plasticity domain where the shear modulus $\mu^{\prime}$ is defined by (3).

$$
\begin{array}{ll}
\rho<\mathrm{R}_{\mathrm{a}} & \tau(\rho)=\mu_{\mathrm{a}} \cdot \psi \cdot \rho \\
\rho>\mathrm{R}_{\mathrm{a}} & \tau(\rho)=\left(\mu_{\mathrm{a}}-\mu^{\prime}\right) \cdot \psi_{\mathrm{s}} \cdot \mathrm{R}+\mu^{\prime} \cdot \psi \cdot \rho
\end{array}
$$

In this range of twist angle, relation (6) gives:

$$
M_{t}=M_{t s} \cdot\left[\frac{\mu^{\prime}}{\mu_{\mathrm{a}}}\left(\frac{\psi}{\psi_{s}}-\frac{\psi^{3}}{\psi_{s^{3}}}\right)+\frac{4}{3}\left(1-\frac{\mu^{\prime}}{\mu_{\mathrm{a}}}-\frac{\psi_{\mathrm{s}}^{3}}{\psi^{3}}+\frac{\mu^{\prime}}{\mu_{\mathrm{a}}} \cdot \frac{\psi_{\mathrm{s}}{ }^{3}}{\psi^{3}}\right)+\frac{\psi_{\mathrm{s}}{ }^{3}}{\psi^{3}}\right]
$$

where $\mathrm{M}_{\mathrm{tS}}$ denotes the critical twisting moment of transformation defined as a function of $\psi_{\mathrm{s}}$ using classical elastic relations: $\quad M_{\mathrm{ts}}=\frac{\mathrm{SR}^{2}}{2 \mu_{\mathbf{a}}} \psi_{s}$

- When $\Psi>\Psi_{\mathrm{f}}$, three domains are to be considered (fig. 3c). From a fatigue limit observation in superelastic loading on SMA it can be considered that $\psi>\psi_{\mathrm{f}}$ has no practical interest for superelastic applications, because the strain in the material is then too important $(\varepsilon>3 \%)$. In the following, this range of twist angle is no longer taken into consideration.

For $\Psi<\Psi_{\mathrm{f}}$, the torque $\mathrm{M}_{\mathrm{t}}$ against rotation $\psi$ is represented at figure 4. Despite the simple bilinear form chosen for the material behavior, this relation is complex due to structure effect. Nevertheless, a bilinear approximation ct the $M_{t}(\Psi)$ relation can be established considering $\left(\frac{\psi_{s}}{\psi}\right)^{3}$ as negligible behind $\frac{\psi_{s}}{\psi}$ in (9), 
and with the definition of a new transformation critical torque $M_{\mathfrak{l}}^{\prime}=\frac{4}{3} \mathrm{M}_{\mathrm{ts}}$. With these assumptions when $\psi>\frac{4}{3} \psi_{s},(9)$ turns into

$$
\mathbf{M}_{\mathrm{t}}=\mathbf{M}_{\mathrm{t}}^{\prime}\left[\frac{\mu_{\mathrm{a}}-\mu^{\prime}}{\mu_{\mathrm{a}}}+\frac{3}{4} \frac{\mu^{\prime}}{\mu_{\mathrm{a}}} \frac{\psi}{\psi_{\mathrm{s}}}\right]
$$

Relation (10) gives a very practical approximation for the calculus of cylindrical bar in torsion.

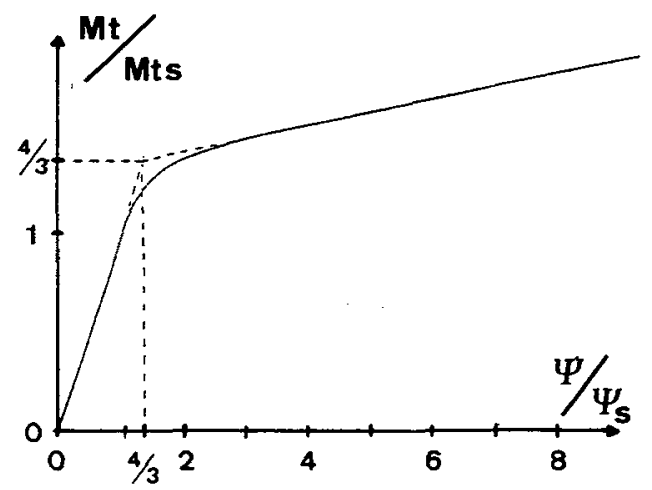

Figure 4: Evolution of the applied torque in function of the twist angle.

Definition of the apparent critical torque of transformation $\mathbf{M}_{\mathbf{t}}$

\section{4. - Application to the helical spring. Load-deflection relation.}

This analysis is now applied to an helical spring. In this case, the bending moment and the tensile force are assumed to be negligible considering the thread of the spring is weak. The shear force compared to the twisting moment $\mathrm{M}_{\mathrm{t}}$ is neglected too. Thus there is a pure torsional shear stress $\tau$ in the cross section of the wire and relations established for pure torsion can be applied. The specimen is loaded by a strength $\mathrm{F}$ at constant temperature $\mathrm{T}$. Denotiong $\mathrm{D}$ the mean spring diameter, the twisting moment is defined by

$M_{t}=F \frac{D}{2}$. Two method of calculus for the deflection, denoted by $f$, are presented.

\section{1. - Complementary-energy method.}

The Crotti-Engesser theorem says that the partial derivative of the complementary energy $\mathrm{W}^{*}$ with respect to load $F$ is equal to the corresponding displacement $f$. According the summation convention for subscripts, the complementary energy density is defined as follows [8]

$$
\frac{\mathrm{d} \delta \mathrm{W}^{*}}{\mathrm{dV}}=\varepsilon_{\mathrm{ij}} \cdot \delta \sigma_{\mathrm{ij}}
$$

For pure twisting, this relation can be replaced by :

$$
\frac{\mathrm{d} \delta \mathrm{W}^{*}}{\mathrm{dV}}=\psi \cdot \rho . \delta \tau(\rho)
$$

Denoting by $\mathrm{n}$ the number of active coils, integration of expression (12) on the whole volume leads to

$$
\delta W^{*} \approx n . \pi \cdot D \cdot \psi \cdot \delta M_{t}
$$

Applying to relation (13) the Crotti-Engesser theorem, it gives :

$$
\delta \mathrm{f}=\frac{\partial \delta \mathrm{W}^{*}}{\partial \mathrm{F}} \approx \mathrm{n} \cdot \pi \cdot \mathrm{D} \cdot\left[\psi \cdot \frac{\partial \delta \mathrm{M}_{\mathrm{t}}}{\partial \mathrm{F}}+\delta \mathrm{M}_{\mathrm{t}} \cdot \frac{\partial \psi}{\partial \mathrm{F}}\right]
$$


Relation (14) leads to a very useful relation using critical torque $\mathrm{M}_{\mathrm{t}}$ and expression (10) previously established for pure torsion. Two cases are then to be considered :

$-\psi<\frac{4}{3} \psi_{s}$ : the applied torque is not sufficient to induce the transformation in a significant way, in that case the classical formulae used in mechanical engineering is obtained from (14). This leads to define a critical elastic displacement $f_{S}$ which is related to the force $F_{S}$ at which $\psi$ equals $\psi_{s}$ (related to the temperature):

$$
\mathrm{f}_{\mathrm{s}}=\frac{2}{3} \frac{\mathrm{n} \mathrm{D}^{3}}{\mu_{\mathrm{a}} \mathrm{R}^{4}} \quad \mathrm{~F}_{\mathrm{s}}
$$

$-\frac{4}{3} \psi_{\mathrm{s}}<\psi<\psi_{\mathrm{f}}$, phase transformation occurs in the wire.

The rotation $\psi$ is obtained from (10):

$$
\psi=\psi_{\mathrm{s}} \cdot\left[\frac{\mu_{\mathrm{a}}}{\mu^{\prime}} \cdot \frac{\mathrm{F}}{\mathrm{F}_{\mathrm{s}}}-\frac{4}{3} \frac{\mu_{\mathrm{a}}-\mu^{\prime}}{\mu^{\prime}}\right]
$$

and relation (14) gives the following expression for the displacement $f$ :

$$
\mathrm{f}=\mathrm{f}_{\mathrm{s}}\left[\frac{4}{3}+\frac{\mu_{\mathrm{a}}}{\mu^{\prime}}\left(\frac{3}{4} \cdot \frac{\mathrm{F}}{\mathrm{F}_{\mathrm{s}}}-1\right)\right]
$$

This method gives relatively good approximations for the spring and has the advantage to be very easy to use but it can be hardly installed on computers for general problems due to the fact that it uses derivation. For computation, another method based on Bresse integrations is more convenient. In addition, this approach gives more detailled informations (rotations, lateral displacements, changes in the geometry).

\section{2. - Method based on Bresse integrations [9]}

Due to the inelastic behavior of SMA, the Bresse integrations are solved using an incremental method . Each step, $\Delta \psi$, of the twist angle $\psi$ induces an incremental displacement caracterised by $\Delta u_{1}$ in translation and $\Delta \omega_{1}$ in rotation.

Increase in $\psi$ is related to variation in $\mathrm{M}_{\mathrm{t}}$ denoted $\Delta \mathrm{M}_{\mathrm{t}}$, in respect with the heterogeneous distribution of shear stress in cross section (fig.3), it comes :

$$
\begin{array}{ll}
* \psi<\psi_{\mathrm{s}} & \Delta \psi=\frac{2 \Delta \mathrm{M}_{\mathrm{t}}}{\pi \cdot \mu_{\mathrm{a}} \cdot \mathrm{R}^{4}} \\
* \psi_{\mathrm{s}}<\psi<\psi_{\mathrm{f}} & \Delta \psi=\frac{2 \Delta \mathrm{M}_{\mathrm{t}}}{\pi\left[\mu_{\mathrm{a}} \cdot \mathrm{R}_{\mathrm{a}}^{4}-\mu^{\prime}\left(\mathrm{R}^{4}-\mathrm{R}_{\mathrm{a}}^{4}\right)\right]}
\end{array}
$$

For the whole spring, the following expressions are finally obtained:

$$
\Delta \mathrm{u}_{1}=\left[\begin{array}{c}
\mathrm{n} \cdot \mathrm{p} \cdot \mathrm{D} \cdot \Delta \psi \\
\mathrm{p} \cdot \mathrm{n} \cdot \frac{\mathrm{D}}{2} \cdot \Delta \psi \\
\mathrm{n} \cdot \pi \cdot \frac{\mathrm{D}^{2}}{2} \cdot \Delta \psi
\end{array}\right](\mathrm{o}, \mathrm{x}, \mathrm{y}, \mathrm{z}) \quad \Delta \omega_{1}=\left[\begin{array}{c}
-(\mathrm{n}-1) \cdot \mathrm{D} \cdot \Delta \psi \\
0 \\
(\mathrm{n}-1) \cdot \mathrm{p} \cdot \Delta \psi
\end{array}\right](\mathrm{o}, \mathrm{x}, \mathrm{y}, \mathrm{z})
$$

in which $\mathrm{p}$ denotes the thread of the spring, and $\mathrm{D}$ denotes the mean spring diameter. 


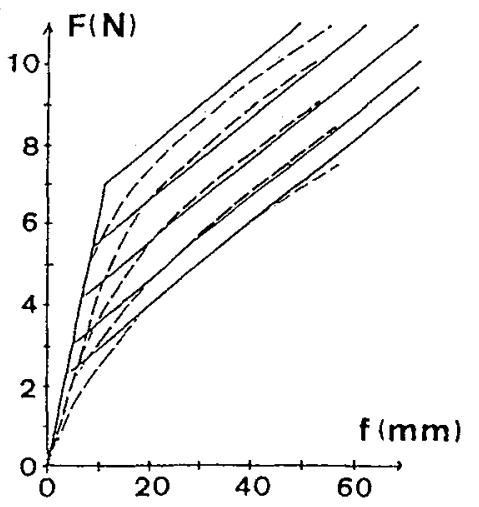

Figure 5: Comparison between experimental and numerical results on a $\mathrm{Cu}-\mathrm{Zn}$-Al helical spring $\left(\mathrm{M}_{\mathrm{S}}=7^{\circ} \mathrm{C}\right)$ for the complementary energy method.

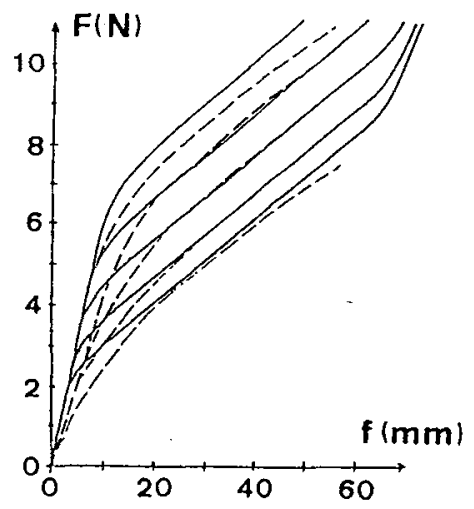

Figure 6:Same comparison for the Bresse integration.

Results obtained for helical spring made of $\mathrm{Cu}-\mathrm{Zn}-\mathrm{Al} \mathrm{SMA}$ are presented in figure 5 and 6 . The geometrical parameters of the spring are : 25 active coils of $1 \mathrm{~mm}$ diameter each with a $5 \mathrm{~mm}$ mean diameter.The material used is a commercial $\mathrm{Cu}-\mathrm{Zn}$-Al SMA elaborated by TREFIMETAUX ( $\left.\mathrm{Ms}=7^{\circ} \mathrm{C}\right)$. Load-deflection curves for this spring are given in figure 1 at different temperatures.

\section{5. - Discussion.}

Both mechanical engineering approaches developed here need only tensile test experiment to characterize the SMA behavior.Material parameters leading to results shown in figure 5 and 6 are determined from measurement on the stress-strain curve presented in figure 2 (experimental shear modulus of transformation $\mu^{\prime}=2600 \mathrm{MPa}$ ).

Comparison between experimental and numerical results shows a good agreement for the two methods (fig. 5 and 6). Relation (17) gives in a very practical form a first approximation for spring mechanical design. The use of Bresse integrations approach leads to a more accurate description of the structure effect (see fig. 6) because no approximation was made in the calculation scheme. This last method is well adapted to micro-computer use.

\section{REFERENCES,}

1. K. NAKAI, T. OKUDA, H. TANAKA, Proc. SMA'86, Guilin, China (1986) 455-460.

2. T. WARAM, in "Engineering aspects of SMA",T.W. Duerig and al., Ed. Butterworth-Heineman (1990) 234.

3. R. STALMANS, Proc. "Science and Technology of Shape Memory Alloys, Barcelona (1989), 463-481

4. T. ATANACKOVIC, M. ACHENBACH, Continuum Mech. Thermodyn.1 (1989) 73-80.

5. E. PATOOR, A. EBERHARDT, M. BERVEILLER, Rev. Traitement Thermique, 234, 43 (1990).

6. H. TOBUSHI, K. TANAKA, JSME Inter. J. Series 1, 34 (1991) 83-89.

7. E. PATOOR, A. EBERHARDT, M. BERVELLER, Acta Metall., 35 , 11 (1987) 2779.

8. J. GERE, S.P. TIMOSHENKO, "Mechanics of Materials",3rd edition, PWS-KENT Publishing company (boston) (1991).

9. S. LAROZE, J.J. BARRAU,"Résistance des Matériaux et Structures", Masson Ed. (Paris) (1984). 